\title{
How null results can be significant for physics education research
}

\author{
Luke D. Conlin, ${ }^{1}$ Eric Kuo, ${ }^{2}$ and Nicole R. Hallinen ${ }^{3}$ \\ ${ }^{1}$ Department of Chemistry and Physics, Salem State University, Salem, Massachusetts 01970, USA \\ ${ }^{2}$ Learning Research \& Development Center, University of Pittsburgh, \\ Pittsburgh, Pennsylvania 15260, USA \\ ${ }^{3}$ College of Education, Temple University, Philadelphia, Pennsylvania 19122, USA
}

(Received 1 October 2018; published 3 July 2019)

\begin{abstract}
[This paper is part of the Focused Collection on Quantitative Methods in PER: A Critical Examination.] A central aim of physics education research is to understand the processes of learning and use that understanding to inform instruction. To this end, researchers often conduct studies to measure the effect of classroom interventions on student outcomes. Many of these intervention studies have provided an empirical foundation of reformed teaching techniques, such as active engagement. However, many times there is not sufficient evidence to conclude that the intervention had the intended effect, and these null results often end up in the proverbial file drawer. In this paper, we argue that null results can make significant contributions to physics education research, even if the results are not statistically significant. First, we review social science and biomedical research that documents widespread publication bias against null results, exploring why it occurs and how it can hurt the field. We then present three cases from physics education research to highlight how studies that yield null results can contribute to our understanding of teaching and learning. Finally, we distill from these studies some general principles for learning from null results, proposing that we should evaluate them not on whether they reject the null hypothesis but according to their potential for generating new understanding.
\end{abstract}

DOI: 10.1103/PhysRevPhysEducRes.15.020104

\section{INTRODUCTION}

Which research findings are significant enough to be published? We propose a simple criterion: Findings are significant insofar as they advance the state of existing knowledge. We suspect that this criterion is general enough that most researchers, regardless of discipline or methodology, could likely agree with it in principle. Of course, there could be many ways to advance the state of existing knowledge in practice.

In quantitative research, one approach to advancing the state of existing knowledge is to establish associations between measured quantities. Regardless of discipline, finding such associations can help researchers understand the mechanisms underlying the phenomena of study, allowing for empirically founded descriptions and predictions. In fields like medicine, psychology, and education, establishing such associations quantitatively is often done through null-hypothesis significance testing, which commonly uses a critical probability value ( $p$ value) to decide whether or not observed associations likely arose by

Published by the American Physical Society under the terms of the Creative Commons Attribution 4.0 International license. Further distribution of this work must maintain attribution to the author(s) and the published article's title, journal citation, and DOI. chance. Research studies showing that the associations between quantities were unlikely to have arisen by chance (a criterion commonly set at $p<0.05$ ) categorize these associations as statistically significant. Studies that fail to establish statistically significant associations are typically considered "null results."

Although finding statistically significant associations has advanced understanding in these fields, researchers often make a critical error by deciding that null results do not advance the state of existing knowledge. At times, when researchers play the role of journal editor or reviewer, they may use the criterion of statistical significance to reject manuscripts containing null results. In their role as authors, researchers can also self-censor their own work, placing null results into a file drawer never to be disseminated. Recently, empirical studies have found that both of these scenarios have contributed to a widespread "publication bias" against null results [1,2], which at best limits the conclusions of the field and at worst brings many of its findings into question [3].

As an interdisciplinary science, physics education research (PER) incorporates research methods as well as results from many fields, including fields where this bias against null results has been shown to exist. Without critically examining these methods and results from other disciplines, PER is at risk of absorbing these threats to research validity. The purpose of this article is to examine 
the bias against null results and to offer suggestions for how researchers can avoid falling into this counterproductive bias. Using three illustrative case studies from our own work, we provide concrete examples of how null results can advance the state of existing knowledge in educational research. We distill several principles for designing and identifying research that produces useful null results, although the principles apply more generally than just to null results. We propose that learning how quantitative null results can advance the state of existing knowledge is part of learning quantitative research methods. We hope this discussion will provide useful guidance for researchers (acting as authors or as reviewers) in assessing the scientific significance of quantitative research findings, regardless of their statistical significance.

\section{WHAT ARE NULL RESULTS AND WHAT DO WE DO WITH THEM?}

\section{A. Learning from null results in physics: The Michelson-Morley experiment}

To illustrate what a null result is and how it can advance the state of current state of knowledge, we turn to one of the most influential experiments in the history of physics: the Michelson-Morley experiment. In 1887, Michelson and Morley [4] published their attempt to measure the relative motion between Earth and the luminiferous ether, the theoretical medium through which light was presumed to travel. The prevailing belief of the time was that the ether was stationary in space as Earth moved through it. This could provide a reference frame with which to measure the absolute velocity of Earth, since the speed of light measured by an observer on Earth would be transformed due to that relative motion: light traveling parallel to the orbital velocity of Earth would appear slower, and light traveling antiparallel to the orbital velocity would appear faster. Using an interferometer, Michelson and Morley measured the phase shift between two beams of light traveling in perpendicular directions to determine the effect of this relative motion and find supporting evidence for existing ether models. Although they made these measurements at several different angles and different times of day, they did not find the predicted effect. They concluded that their measured phase shift was probably less than $1 / 40$ th of the predicted value based on the properties of the experimental apparatus and the known orbital velocity of Earth. Despite their experimental setup having more than enough sensitivity to measure the predicted effect, Michelson and Morley did not find it; their experiment yielded a null result.

Although counter to their predictions, Michelson and Morley published these results, pointing out that, if true, their findings contradicted the dominant theoretical models of light at the time. They also proposed an alternative hypothesis that would explain why their experiment did not match the prediction: the motion of the solar system, which was not taken into account in their design, could have been exactly right at the time of the experiment to cancel out the motion of Earth relative to the solar system. Although they argued that this explanation seemed unlikely, they proposed to repeat the experiment every three months to rule out this explanation. Repeated experiments ultimately failed to find the hypothesized effect. Michelson and Morley's null result went on to provide an empirical basis for Einstein's special theory of relativity, which rejected the existence of the ether and the very notion of absolute velocity.

Michelson and Morley's experiment provides a model for how to handle a null result in a way that advances the state of existing knowledge in a research field. The value of this experimental result was not that it matched the predicted effect but in how it fit coherently (or not) with existing theories and empirical results. By analogy, physics education research can similarly benefit from welldesigned studies that produce null results.

\section{B. Null results in quantitative education research}

Like physics research, quantitative education research often seeks to establish specific associations between measured quantities. To that end, quantitative education research relies heavily on null hypothesis significance testing (NHST). In the formal logic of NHST, the researcher's stated hypothesis (e.g., that two quantities are associated) is contrasted against a null hypothesis (i.e., that there is no association). The empirical evidence is then evaluated in light of the null hypothesis, using statistical tests to decide the likelihood that any measured association arose due to random sampling (sampling error) from a null distribution. For example, in the context of a physics course, a researcher might hypothesize that students' surveyed expectations toward learning physics would positively correlate with their course grade. In this case, they would compare their measured correlation against the null hypothesis, which would predict zero correlation between expectations and course grade. Statistical tests can compute the probability (or $p$ value) that the observed results or even more extreme results would have been observed if the null hypothesis were true. For example, a $p$ value of 0.01 indicates that there is a $1 \%$ chance that the observed results (or even more extreme results) would have been observed from random sampling of a null distribution. It is conventional in many social sciences and educational research fields to use a $p$-value cutoff level of $p<0.05$ (which would be described as a $2 \sigma$ difference in physics) to categorically decide whether the observed results were likely or unlikely to have come from the null hypothesis. If the probability is categorized as unlikely, then the researchers "reject" the null hypothesis and often consider their original hypothesis to be confirmed: there exists a "statistically significant" association 
between quantities. ${ }^{1}$ If, on the other hand, the $p$ value does not meet the cutoff $(p \geq 0.05)$, there is not sufficient evidence to reject the null hypothesis. This is considered a null result.

NHST can be a powerful tool for researchers to advance the state of knowledge, by establishing associations between measured quantities (or lack thereof). This can be accomplished regardless of whether the $p$ value makes the cutoff. Just as Michelson and Morley's null result contributed to advancing the state of physics knowledge, we contend that null results in fields that use NHST have the possibility to advance the state of knowledge in those disciplines.

In the disciplines that use NHST, there is a relative absence of null results in the literature that suggests that their potential to contribute to scientific knowledge is not generally appreciated. Large metastudies of prominent psychology and biomedical journals have found that among articles that use tests of significance, almost all (>95\%) reject the null hypothesis $[3,6]$. The striking absence of null results in the published literature is suggestive of a widespread bias against null results. We now consider the evidence of this bias, its potential causes, its negative consequences for research, and the risks it poses to PER.

\section{Publication bias against null results and its consequences}

There have long been concerns among social scientists that null results are more likely to be "filed away" rather than published, which would mean that published studies represent a biased sample [7,8]. As Rosenthal [7] put it, the extreme version of this "file drawer" problem would be that the published literature consists only of type-I errors, i.e., studies whose $p$ values were less than 0.05 due to chance and therefore incorrectly rejected the null hypothesis. Although this extreme outcome is unlikely, it is difficult to determine the full extent of this publication bias, since a full accounting would need to determine the number of unpublished null results.

Early attempts to estimate the extent of publication bias in psychological research found that doctoral theses and conference abstracts contain significantly higher proportions of null results than published journal articles [6,7]. More recent experimental approaches have increased

\footnotetext{
${ }^{1}$ Cohen has discussed the logical fallacy of the common practice of using null hypothesis significance testing to reject the null hypothesis and confirm the original hypothesis [5]. The crux of the issue is that (i) NHST indicates how likely it is for the observed data to have come from the null hypothesis, not how likely the null hypothesis is given the observed data and (ii) there exist many possible alternative hypothesized mechanisms that could lead to the same results. Although we recognize these flaws with null hypothesis significance testing, our critiques in this paper are largely independent of this issue, focusing on other issues with how NHST is used.
}

transparency by subjecting research methods to peer review before data are collected, which allows for a more careful accounting of which research results are published. Smyth et al. [9] used clinical trial registries to compare outcomes reported in trial protocols versus those that were published, finding significant evidence of reporting bias. Franco, Malhotra, and Simonovits [1] used preregistered reports in the time-sharing experiments in social science (TESS) to analyze a set of 221 social sciences studies whose fates were known to be either published, unpublished, or not written up at all. They found that studies characterized as having "strong" results (with significant statistical tests supporting hypotheses) were 40 times more likely to be published, and 60 times more likely to be written up than studies with null results.

What causes this publication bias against null results? The fact that TESS authors were less likely to even write up null results suggests at least part of the publication bias is due to authors' self-censoring [1]. Email interviews revealed that these authors self-censored sometimes due to perceived bias in the journals, and sometimes due to their own biases. Some authors reported giving up on publishing because of anticipated editorial pushback due to nonsignificant $p$ values, and some because they simply regarded these studies as failures. Smyth et al. [9] also found evidence that the observed publication bias was partly due to the researchers' own perceptions of null results. They conducted phone interviews with authors, finding their observed reporting bias against null results was generally due to authors' poor understanding of how null results could contribute to the evidence base.

Researchers need a better understanding of how null results can advance the state of current knowledge, and conversely, how neglecting to publish null results can hinder research progress. Next, we look at how neglecting to publish null results can compromise the evidence base in specific ways, before turning to how the field has started to address the issue.

\section{Negative consequences of the publication bias against null results}

The publication bias against nonsignificant findings interferes with meta-analyses, which attempt to synthesize across a representative sampling of results. When rigorously established null results are absent from the literature, meta-analyses will overestimate the magnitude of the effects. This has significant consequences as these metaanalytic results can inform decision making, including decisions in areas such as education and health policies.

Researchers conducting meta-analyses have dealt with these biases in several ways. One is to seek out effects in unpublished papers (such as dissertations) or to even contact researchers in the field to obtain results from unreported studies. Although this does combat the bias against null results to some degree, the success of the 
approach relies on an author's ability to network with researchers and the willingness of these researchers to share their null results. Another approach is to calculate how a number of unpublished null results would affect the results. For example, in a meta-analysis of 158 studies comparing conceptual inventory scores after traditional instructional methods and after active learning in STEM classrooms [10], active learning was found to produce higher scores on exams and concept inventories by $0.47 \mathrm{SD}$. To investigate the potential effect of publication bias, they calculated the number of studies with an effect size of zero needed to reduce the overall effect size to $0.20 \mathrm{SD}$, finding that 114 such unpublished null results would be needed. Although this provides a useful estimate of how unpublished studies would affect the results, there are unfortunately no clear guidelines to determine what number of unpublished null results plausibly exists.

A second negative consequence of a publication bias against null results is that it contributes to an emerging "replication crisis." Recently, fields such as psychology and cancer research have found that results believed to be empirically established are not replicable [11,12]. There have been arguments that the implicit goals of establishing new associations between quantities in these research professions have discouraged replication attempts, and that over-reliance on statistical significance has overestimated the certainty of significant effects and underestimated the status of null findings [13]. Because the culture of professional science can value innovation over replication, highfidelity replication attempts can be devalued, even if these replication attempts produce null results that challenge existing beliefs. Neuliep [14] found that psychology journal editors largely preferred to publish new results rather than replications. More recently, a survey of 1151 psychology journal policies showed that only 33 (3\%) explicitly encouraged the submission of replication studies [15]. This bias against publishing replications (and, in particular, replication null results that could falsify initial theories) threatens the ability of a research field to falsify theories and truly test ideas scientifically [14].

To counter these biases, recent efforts have explicitly sought to test the replicability of empirical science in these fields. The Open Science Collaborative [11] performed high-quality replication attempts for 100 psychology studies. Only $39 \%$ of these attempts were rated to have replicated the original findings. Similarly, The Reproducibility Project: Cancer Biology, which is currently in progress, has aimed to replicate a series of high-profile studies. Twelve of the replication studies are currently published: 4 have reproduced key parts of the original studies, 4 have reproduced key parts of the original studies with some additional results that were inconsistent or uninterpretable, 2 failed to reproduce the findings of the original studies, and 2 could not be interpreted [16]. These recent efforts have helped drive the ongoing search for ways to overcome a bias against null results to improve the validity and reliability of the knowledge produced in these fields.

\section{Attempts to counteract the publication bias}

While concerted attempts to replicate findings helps address the replication crisis, they still do not address a root cause - the widespread conflation of statistical significance with scientific significance. Many quantitative methodologists have pointed out that overemphasis on $p$ values stems from a widespread misunderstanding of their meaning and relative importance $[5,17]$. The perceived objective certainty of NHST may contribute to overvaluing of $p$ values, whereas in truth no one study's $p$ value can confirm or deny a theory [18]. Additionally, the $p$-value cutoffs are used to arbitrarily make dichotomous judgments about a continuous probability distribution. As Rosnow and Rosenthal [18] put it: “...surely, God loves the .06 nearly as much as the .05. Can there be any doubt that God views the strength of evidence for or against the null as a fairly continuous function of the magnitude of $p$ ?" (p. 1277). The arbitrary nature of "what counts as statistically significant?" is also made obvious by pointing out that other fields use different $p$-value cutoffs to decide what is significant, like particle physics which uses criteria of $p<.003(3 \sigma)$ for "evidence of a particle" and $p<.0000006(5 \sigma)$ for "discovery of a particle."

Methodologists have begun to point out this confusion, and in doing so have begun to issue guidelines of how to better statistically gauge the scientific significance of research results $[5,17,19,20]$. For example, the American Psychological Association (APA) convened a task force on statistical inference, resulting in a recommendations document [19]. The recommendations downplay the importance of $p$-value tests of statistical significance. Instead, they recommend assessing practical significance by reporting other more informative statistics such as effect sizes and confidence intervals. To address generalizability, they recommend conducting replication studies.

While these recommendations represent good first steps toward countering the misinterpretation of $p$ values in quantitative research, they do not adequately address the potential contributions of null results. For instance, the APA recommends supplementing $p$ values with reporting of effect sizes, but they do not address how a study can contribute to the literature even if the effect size is small [19]. We argue that guidelines on quantitative research methods must also include guidance on what to do when the statistical tests come up negative. This article represents an attempt to take some first steps in this direction. We hope the points made here will help researchers assess the significance of their own and others' research regardless of the statistical significance. We first consider the threats the publication bias against results poses for PER, before 
introducing some specific ways that null results can advance the state of knowledge in educational research.

\section{Assessing the risks of publication bias in PER}

To our knowledge, no systematic analysis of the publication rates of null results and replications in quantitative research studies has yet been conducted for PER, so we do not know if a publication bias against null results exists. However, as an interdisciplinary endeavor, PER does draw on the quantitative research methods from other fields, as well as some of their findings. As such, we argue that one risk of adopting these quantitative methods is adopting the mindsets overvaluing statistically significant results that lead to the bias against null results seen in other fields.

Assessing the risks of a bias against null results in PER raises a number of questions. First, would a publication bias greatly impact existing research efforts in PER? As discussed previously, publication bias against null results presents challenges for meta-analysis and replication, and we discuss the role of each of these in PER. On meta-analysis: physics education researchers (and discipline-based education researchers more generally) have conducted large-scale meta-analyses that consolidate our knowledge and propel reform efforts. These meta-analyses span several topics related to learning and instruction, including active learning [21-23], as well as learning attitudes and epistemologies [24]. A publication bias against null results would lead to overestimates of these metaeffects.

Replication is also an especially salient goal for PER as a discipline which at times aims to disseminate and replicate the efficacy of instructional reforms across a variety of contexts. There is not currently a clear replication crisis in PER, but there is evidence that the research to date has focused on narrow, nonrepresentative samples [25]. This raises questions about whether what we know about physics teaching and learning reliably replicates across instructional contexts and student demographics. If future replication attempts yield null results, a potential bias against null results could bury these findings, masking the contextual variability of the initial findings. A second question is, what underlying commitments or goals in PER could buffer against a null result bias? We pose that one such commitment is that PER seeks knowledge that is instructionally significant - by which we mean both knowledge that leads to practically meaningful differences in instruction and knowledge that plausibly fits in with and contributes to instructional practice. Whereas incentives and goals in other fields may push focus toward statistical significance, PER's roots in instructional practice may provide an instructional counterperspective to purely statistical considerations. This may help lessen the importance placed on statistically significant results. Relatedly, PER is a mixed-methods field, incorporating understanding from qualitative observation and quantitative results. Qualitative observations of student thinking and classroom activity may provide another lens for evaluating the validity and significance of research findings that counters purely quantitative interpretations.

Finally, what underlying commitments or goals in PER could increase the risk of null result bias? Because physics education researchers sometimes aim to establish the effectiveness of novel teaching methods, these researchers may be reluctant to publicize failures to replicate the efficacy of these instructional innovations. Although there is much empirical evidence in favor of many PER-based instructional reforms, null results may complicate the story. When convincing resistant instructors or institutions to change, physics education researchers may fear that resistant faculty may use such null results to justify their refusal to change their instructional approach.

Independent of whether these risks are currently realized in PER, it is clear that adopting the quantitative methodologies from social science fields introduces the risk that we will also inherit the biases and subsequent problems that have arisen from these methodologies. When doing quantitative research, it is easy to fall into the trap of treating null results as experimental failures. Additionally, guidelines on quantitative methodologies often fail to articulate how null results can help advance understanding in a field. To clarify how studies that fail to establish associations between measured quantities can contribute to shared knowledge, we give three examples from our own work of how null results raised new questions and new directions for research when situated within existing findings in the research literature.

\section{HOW PUBLISHING NULL RESULTS CAN CONTRIBUTE TO KNOWLEDGE IN EDUCATION RESEARCH}

Using examples from our own work, we illustrate three ways that null results can advance existing knowledge, even without establishing statistically significant associations between quantities. We do not mean to suggest that these three examples illustrate the complete set of ways that null results can contribute to advancing the state of existing knowledge; we present these examples as three case studies of how researchers in PER can learn from null results.

Specifically, we present cases that highlight three potential contributions of null results: (1) providing existence proofs of alternative approaches, (2) illustrating failures to generalize, and (3) showing replication failures.

\section{A. Case study 1: Null results can provide an existence proof}

Quantitative studies in PER often compare a novel teaching approach to an already established approach. Even when the measured outcomes produced are not measurably different than current approaches, research 


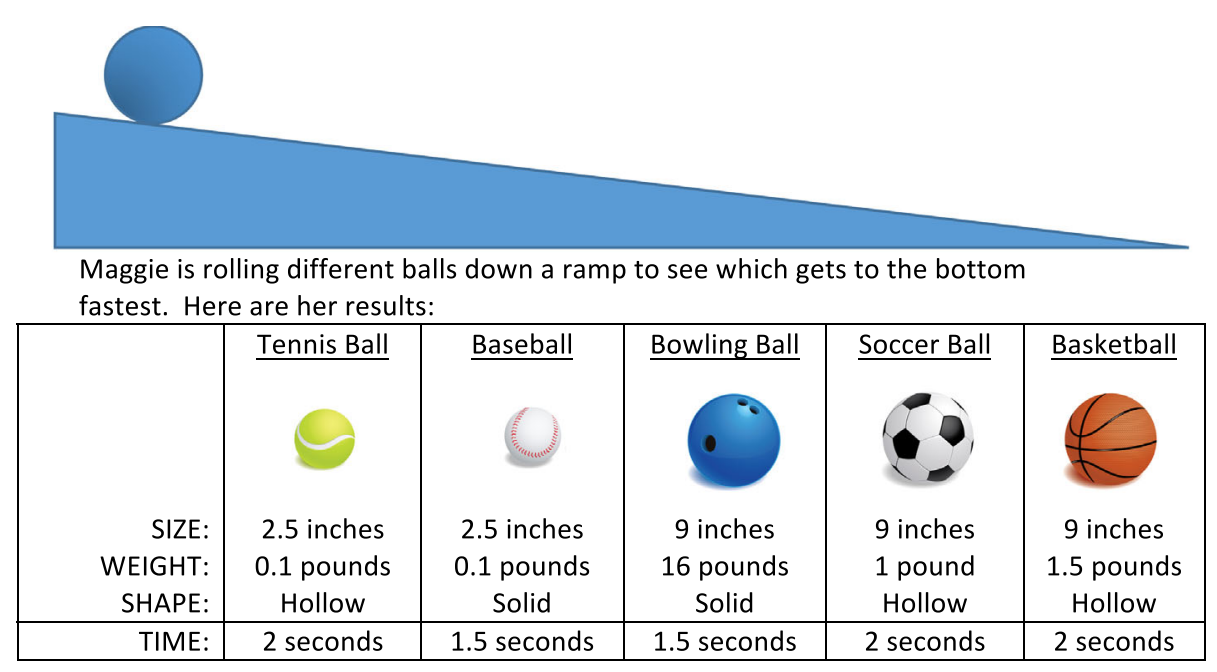

FIG. 1. The "ramp" post-test item. In two questions, students were asked to say which balls they would compare and to see if they could figure out what makes a difference in how fast a ball reaches the bottom of the ramp.

producing null results can still help advance existing knowledge by providing an existence proof that a novel alternative approach may be viable. This was the case in a study by Conlin, Hallinen, and Schwartz [26], which compared how middle school students learned and used a new strategy for conducting scientific inquiry.

Studies of learners' inquiry into related variation are concerned with their skills at making valid comparisons in the data to discern causal factors. In these studies, one deductive strategy for making comparisons has dominated research and instruction - the control-of-variables strategy (CVS). CVS involves making case comparisons that differ on only one independent variable, to see whether that independent variable has an effect on the dependent outcome. Many studies of students' inquiry have demonstrated that CVS can be taught, and that using the strategy can improve students' performance in determining causal relationships through data analysis [27,28]. However, controlling variables to deduce causes is not the only way scientists make fair comparisons with data. Often such comparisons are made inductively, comparing cases with the same dependent outcome to see if the independent factors that cause that outcome can be determined. This is often the approach in cases when the independent variables cannot be manipulated at will, for instance, in medical diagnosis or astronomical observations. Conlin, Hallinen, and Schwartz proposed this inductive strategy, which they called the general principle strategy (GPS), as an instructional alternative to controlling variables.

The hypothesis was that students who were taught GPS would be better prepared to discover multivariate relations than students who were taught CVS. Although CVS is useful for discovering reliable single-variable dependencies, it is not well suited for discovering relations that may only be detectable when multiple quantities are changed at once, since students would have to systematically vary multiple variables one at a time and synthesize the results across many cases in order to find the multivariate pattern. For instance, students using a CVS strategy to investigate the factors that affect how much a spring stretches when a mass is hung from it may systematically vary the length of the spring, the coil diameter of the spring, or the hanging mass one at a time. However, these students may have difficulty finding multivariate effects, such as how the precise effect of mass on spring stretch depends on the width of the spring, when only changing one variable at a time. GPS may offer a clearer route to discovering multivariate relations, by comparing across common dependent outcomes (same amount of spring stretch) and finding how different combinations of the independent variables can yield the same result.

To test the hypothesis that GPS would help students detect multivariate relations better than CVS, Conlin, Hallinen, and Schwartz conducted a study in five middle school science classrooms. Students $(n=100)$ were randomly assigned into one of two instructional conditions, either general principle (GP) or control-of-variable (CV) instruction. Over seven class periods, students in each condition were taught their respective inquiry strategy (GPS or CVS) and were taught to apply the strategy in investigating relations for a series of physics topics (projectiles, pendulums, and collisions). In the lessons, students searched for relationships between physical properties through inquiry in hands-on experiments as well as computer-based physics simulations.

To test the hypothesis, two assessments measured which strategy students used to explore data in determining how the independent variables affect the dependent outcome. The ramp posttest item presented five cases of balls rolling down a ramp. Each case could differ on the dependent outcome, time to reach the bottom, and three independent variables, size (diameter), weight, and shape (hollow or 
solid) (Fig. 1). Students were asked to (1) choose which cases to compare to determine which factor affects the time to roll down the ramp and (2) pick which factors matter, based on those cases. The cases chosen were used to classify students' strategies: GPS (selecting cases with the same outcome), CVS (selecting cases that only differed on one independent variable), or neither strategy. Counterintuitively for students, the shape of the ball (hollow or solid) was the only factor that affected the time it took to roll down the ramp.

The second assessment was the "balancing act" posttest. Here, students explored the relationship between torque, mass and lever arm using a computer simulation in which they could place masses on either side of a seesaw at different distances from the fulcrum [29]. When they were ready, students could choose to enter a challenge mode where they were asked to predict which way different configurations of weights would make the balance beam tip. Although students could advance through the initial levels of the challenge mode with only knowledge of single variable effects (e.g., greater mass means more torque; greater distance from the fulcrum means more torque), the most difficult questions required students to discover the precise quantity for torque $(m \times d)$ to accurately predict the direction of tipping. In the challenge mode, students answered progressively more difficult questions until they got one wrong or correctly answered eight in a row. The number of questions correctly answered in the challenge mode was used as a measure of how precisely students were able to discover the multivariate relation of torque.

This research study yielded a null result: on average, there was no statistically significant difference in students' ability to detect the correct relationships on the ramp and balancing act posttest whether they experienced the $\mathrm{CV}$ or GP instruction. Although the core prediction turned out to be a null result, many subsidiary predictions of the study did establish GPS as a potentially useful instructional strategy. First, GPS was teachable. On the ramp posttest, there was an association between the instruction experienced (GP or CV) and which strategies students used to make comparisons (GPS, CVS, or Neither). For instance, $28 \%$ of students who were in the GP instruction condition used the general principle strategy, compared to only $2 \%$ who were in the CV instruction condition. Second, GPS and CVS were both more effective than not using any strategy. When comparing students according to which strategy they used on the ramp posttest, there was no statistically significant difference between CVS and GPS use for finding the correct relationship between variables, on either the ramp posttest or balancing act posttest. However, in both cases, use of either strategy was more effective than no strategy.

Although an experimental study of the difference between GP and CV instruction on students' ability to discover multivariate relationships produced a null result, the results help establish the potential use of a new strategy, the general principle strategy, in teaching science inquiry. Given the prevalence of CVS as an instructional inquiry strategy, it is useful to compare its efficacy to alternatives. These results established the viability of GPS as a potential alternative to the ubiquitous control-of-variables strategy and could provide an initial step towards finding areas where GPS can be more effective than alternative strategies. They also provided a potential explanation for why the hypothesized benefit of GPS was not found here: because few students took up GPS (only 15 out of all 100 students in the experiment), it could be that the demonstrable efficacy of the strategies might depend on the effectiveness of the GP instruction. One future direction proposed for revising this study was to enhance the GP instruction, so that more students take up GPS.

\section{B. Case study 2: Null results can show how effects fail to generalize to new contexts}

Null results can also contribute to new understanding if they show how established effects can fail to generalize to new contexts. In a recent example from the medical research literature, Milam, Cohen, Mueller, and Salles [30] investigated gender stereotype threat in female surgical residents. Stereotype threat occurs when a person in a stereotyped group feels at risk for conforming to those stereotypes. A well-established version of the stereotype threat effect is that performance can be lowered by reminding the members of the stereotyped group that they are expected to perform worse. Even though a stereotype that women are worse surgeons exists, the standard stereotype threat induction did not lower female surgical residents' performance on mental rotation or working memory tasks. To explain this finding, they proposed that, to reach a high level of achievement in the face of a negative stereotype, female surgical residents may be selected to be resilient to stereotype threat, either intrinsically or by developing adaptive strategies to mitigate stereotype threat. More generally, finding that widely established effects may not generalize to a novel population helped researchers recognize potential bounds of a phenomenon and lead to new hypotheses with the potential to deepen understanding around a familiar finding.

In an example from our own work on students' problem solving, Hallinen and Booth (under review) sought to extend existing findings on a mathematical problemsolving effect to a new class of problems. Previous research on mathematical problem solving has revealed that the format of problem presentation has an impact on students' problem-solving accuracy: students solve simple algebra problems more accurately when they take the form of a word problem rather than equivalent algebraic expressions to be solved [31]. This finding countered the dominant instructional common sense, that symbolic algebra methods should be mastered first before being applied on more 
difficult word problems. The explanation for this counterintuitive finding was that even students who had not yet mastered the formal algebraic manipulations could successfully use other, informal strategies, such as guess and test or unwinding, to solve the word problems. In guess and test, students used trial and error, progressively selecting numbers for the unknown value and calculating the result. In unwinding, students worked backwards from the desired value given in the word problem, performing a chain of operations to fill in the necessary steps. Both of these strategies can be correct, but are considered informal because they do not follow a standard, efficient algorithm commonly taught in algebra classes. Researchers found that the increased accuracy on word problems went away for more complex problems; students were more accurate on symbolically presented algebra problems (i.e., equations) than on equivalent word problems when there were more than two steps required to solve the symbolic expressions [32]. In these previous experiments, two possible mechanisms were proposed for this shift with complex problems: (1) the algebraic formalism is more beneficial for more complex problems and (2) students are less likely to use informal solutions when solving complex world problems. Since the original effect was observed for linear algebraic equations, further research on the generalizability of these findings to other problem types could shed light on the underlying mechanisms.

Hallinen and Booth extended this line of inquiry to a new problem type, algebraic proportion problems, investigating whether students would be more accurate when the problem was presented as a word problem or as a symbolic equation. The study involved students enrolled in Algebra I classrooms $(n=351)$ throughout the midwestern U.S. These students completed a variety of mathematics problems during class time. Students completed six types of problems. Three sets of problems were symbolic equations: symbolic simple linear problems (e.g., $x-10=4$ ), symbolic complex linear problems [e.g., $(1 / 2)(x-6)=1-3 x$ ] and symbolic proportion problems (e.g., $4 / 3=8 / x$ ). Students also solved proportion word problems, and completed two types of problems that required students to generate symbolic proportions from word descriptions without solving them, either from word equations (" 6 is to 30 as 5 is to $x ")$ or from word problems. The primary research question was whether students would be more accurate on proportion problems presented as word problems or presented symbolically. If proportion problems are more similar to simple linear equations, then students would be more accurate on the verbal word problems format; if they are more similar to complex linear equations, then students would be more accurate on the symbolic format. Secondary research questions included comparison of the relative difficulty of the three types of symbolic problems (simple linear, complex linear, and proportion) and explored students' problem-solving strategies on the proportions word problems.
The primary finding was a null result: there was no statistically significant difference in accuracy between proportions problems presented symbolically versus as word problems. Students were generally quite accurate on both of these types of problems (81\% correct for proportions equations and $75 \%$ correct for word problems). Although an association between problem type and accuracy was not established, the null result clarified the extent to which previous findings in the field could be applied to other types of problems.

Furthermore, comparison with additional problem types advanced our understanding of what potential mechanisms do (or do not) explain differences in accuracy between different problem formats. Additional analysis showed that accuracy on symbolic proportion and proportion word problems was equal to accuracy on symbolic simple linear equation problems. This finding led to one possible explanation for the null result: although students' performance on symbolic proportion problems was comparable to their performance on symbolic simple linear equation problems, they were not able to reproduce the higher accuracy on the word problem format. A potential explanation is that students may not as readily take advantage of informal strategies on the proportion word problems, either because they do not have effective, informal strategies or because they prefer formal symbolic approaches here. Coding of students' written problem-solving strategies provided support for this explanation by showing widespread use of the standard algorithmic cross-multiplication strategy on both symbolic proportion problems and proportion word problems. Very few students used informal strategies. Contrasting the results of this study to the original findings helped clarify the essential mechanism of the original effect: the availability of informal strategies for word problems. Here, the widespread use of a formal algorithm for proportion problems overshadowed potential informal strategies that could potentially support increased accuracy on proportion word problems.

In this study, the null result for proportion problems was itself surprising. Additional analysis helped generate potential explanations that increased our understanding of not only the null result for proportion problems, but also of the mechanisms underlying the original accuracy differences on word problem and symbolic formats. The results here showed that the original effect depended on the availability and use of effective informal problem-solving strategies, strategies which are not always available and used. Importantly, situating the null result against the backdrop of existing findings helped bound the generalizability of the existing effects. This null result empirically demonstrated that accuracy differences for different problem-solving formats is not a general truth. It also established the problem-solving conditions where we can expect student problem solving will be more accurate on one problem format over another. 


\section{Case study 3: Null results can reveal the necessary conditions for replication}

Although finding generalizable effects is one of the goals of education research, no one research study alone can establish the generalizability of any finding. Replication in different contexts is an important component of research methodologies that seek to establish reliable and useful educational principles. In replication attempts, null results can be a canary in the coal mine, warning of possible overgeneralization of results and motivating efforts to develop a more nuanced understanding of the underlying mechanisms and necessary conditions. This was the case in a study from Kuo, Hallinen, and Conlin $[33,34]$, which set out to replicate a surprising effect of prompting students to draw a diagram during problem solving.

Free-body diagrams are a representational tool commonly used to keep track of the forces acting on a system. In introductory physics, students are commonly taught to use free-body diagrams to formalize and keep track of their reasoning on Newton's laws problems. In this way, freebody diagrams are an instructional problem-solving scaffold that instructors hope support students' burgeoning problem-solving expertise. Heckler [35] investigated the effect of explicitly prompting students to draw free-body diagrams before solving force problems. Undergraduate introductory, calculus-based physics students at a large midwestern university were given two typical force and motion problems. The students were randomly selected either to receive a version of the questions that included a written prompt to draw a force diagram to help solve the problem or to receive no such prompt. By analyzing their written solutions, Heckler found that the free-body diagram prompts both (i) decreased the accuracy of students' problem solving and (ii) pushed students to engage in more formal problem-solving approaches. In alignment with the algebra word problem studies described previously, Heckler proposed that students may be more successful on force problems when using intuitive, informal solutions rather than formally taught ones. Notably, this finding ran counter to the standard instructional wisdom that the diagram prompts would act as a problem-solving hint, helping guide students to the correct problem-solving approaches and correct answer. If this finding is reliable and generalizable, these results could have important implications for how problem solving for force problems is taught.

Kuo, Hallinen, and Conlin [34] attempted to replicate and extend Heckler's study. The participants were undergraduate students in an introductory algebra-based physics course at a large, private university. First, all students were given two force problems. Half of the students were randomly assigned to receive an explicit prompt to draw a free-body diagram before solving each problem; the other half were simply asked to solve the problems. One problem was taken exactly from Heckler's original study. The other problem was new, testing whether the original effects could extend to new problems. As before, the key measures were (i) whether students answered the questions correctly and (ii) whether they used a formal approach or an informal shortcut.

The results partially replicated those of the original study. The effect of the diagram prompts on problem-solving approach were replicated: students who were prompted to draw force diagrams used a more formal problem-solving approach, while unprompted students were more likely to use an informal, but more efficient approach. However, unlike Heckler's original study, we found a null result when examining accuracy: there was no effect of diagram prompts on accuracy. In seeking an explanation for the failure to replicate the effect on accuracy, we compared the percentage of correct final answers on the problem common to both the original and replication study. The accuracy for the prompted students in the original and replication study were comparable ( $40 \%$ and $37 \%$ correct, respectively), but the accuracy for the unprompted, control group was $20 \%$ higher in the original study compared to the unprompted group in the replication study (60\% and $40 \%$ correct, respectively). In sum, in both the original and replication studies unprompted students used more intuitive, informal, and insightful solutions than their prompted counterparts. However, only in the original study were the unprompted students significantly more accurate than students who received an explicit diagram prompt.

With respect to the effect of diagram prompts on accuracy, the replication attempt failed, producing a null result. At the same time, this replication study produced a surprising finding when compared to the original study and allowed us to hypothesize several reasons why the effects of diagram prompts on accuracy did not replicate. An explanation consistent with the results is that students in the original study were more prepared to execute informal solutions to reach the correct answer. Although the lack of the diagram prompt opened up the problem space to more informal and intuitive approaches, these approaches may not lead to success if these informal solutions are not physically valid. One reason for this difference in informal approach success could be the difference between calculusbased physics students in the original study and algebrabased physics students in the replication study. It is common that calculus-based students have a stronger mathematics and physics preparation, and so these students may have been better able to execute valid, informal solutions in the original study. Another possible reason for this difference was the unusually fast pace of the physics course in the replication study, which spent only two 50minute lectures covering force problems. Students may not have received enough practice for the presence or absence of the diagram prompt to impact their accuracy (i.e., students who would solve the problem incorrectly would do so in either condition). Although we do not know the 
exact length of instruction in the original study for comparison, we pose that replication of the accuracy effect here could rely on the length (and quality) of instruction, and that future studies could attend to these dimensions as explanatory in replication (or failures to replicate). That the instructional time was not reported in the original study only illustrates our point that null results can bring attention to new, potentially critical factors not originally considered.

Additionally, the replication study uncovered a possible mechanism for how prompting could lower accuracy. It was found that solution type used affected accuracy, where the formal Newton's second law approach was less accurate than informal alternatives. Most incorrect, formal solutions were incomplete; students knew how to start the Newton's second law approach but did not know which objects in the problem to analyze to solve for the relevant force. Diagram prompts could impact accuracy by more strongly suggesting these formal approaches (i.e., drawing a free-body diagram of an object naturally leads into a formal Newton's second law analysis) to students who do not know how to complete them correctly.

This replication study set out to determine whether a surprising effect could be reproduced in a new context. While one of the two original effects was not replicatedthat prompting diagram construct decreased problemsolving accuracy-comparison to the results in the original study shed light on possible explanations for the discrepancy. The null result both forced us to come up with explanations for the failure to replicate and provided possible explanatory avenues for future research. Importantly, the fact that one of the two original effects was replicated-that prompting diagram construction increased use of formal problem-solving approachessuggested that the null result was not due to a gross, methodological mistake. One conclusion from this study could have been that the null result was due to an incorrect design or execution of the study. The partial replication boosts confidence in the experimental procedure and the validity of the results, further indicating that the null result is a valid failure-to-replicate that must be explained rather than one to be brushed off or ignored.

\section{RESEARCH TO GENERATE NEW UNDERSTANDING: SUGGESTIONS FOR EXPERIMENTAL DESIGN AND EVALUATION}

Quantitative studies are commonly judged by their ability to establish statistically significant associations between measured quantities; we propose an alternative measure of quality: whether the findings have the potential to advance the state of existing knowledge. Using this criteria, even null results can be seen as important research results, especially when considered within the larger scope of related research on a topic. We have presented three ways that null results can contribute to new understanding: by providing existence proofs of the viability of new approaches, by showing how previous results can fail to generalize to new contexts, and by uncovering the necessary conditions for replication. In each case, the findings refine existing theoretical positions. We have chosen these three types of contributions using examples from our own work. We do not want to suggest that these are the only ways that null results can contribute to greater understanding; there may be others.

The criteria for deciding whether research findings can advance the state of existing knowledge is less methodologically clear than deciding whether a computed $p$ value is less than 0.05. Rather than presenting clear, objective, and definite criteria, here we orient researchers to science as seeking coherence. In this mindset, the process of science is the continual building and rebuilding of the most coherent argument that ties together theoretical models and empirical data [36]. In the three examples of null results presented, the results contribute to the state of existing knowledge not by establishing new associations between variables but by building connections to and identifying inconsistencies with what is already known in the field. Research on the general principle strategy demonstrated a novel alternative to the thoroughly investigated control-ofvariable strategy. The findings on algebra and force problem solving were inconsistent with existing findings in the field, demanding the search for new theoretical explanations and/or empirical data for building a coherent understanding of these phenomena.

\section{A. Practical steps for seeking coherence in quantitative research}

A focus on coherence suggests practical steps for quantitative researchers. When forming research designs, including multiple quantitative measures is key for generating the empirical data that guides construction of coherent explanations. Using outcome measures that match those used in previous studies, either matching identically or being similar in principle, can guide cross-study comparisons and explanations. For example, in PER, the widespread use of mechanics concept inventories (such as the FCI and FMCE) and attitudinal or expectation surveys (such as the MPEX and CLASS) have helped researchers compare findings directly across studies in the field. In our work, we measured accuracy and strategy use on algebra and force problems, in part to compare to existing results in the field. In part, the null results we found raised new questions about existing findings, because the types of measures used were so similar.

Additionally, researchers can use these multiple measures to construct coherent hypotheses within their own research studies. When multiple, independent measures all show the predicted effects, the convergence of these measures can provide greater support for the initial hypothesis (this is called convergent validity). When there is inconsistency between these multiple measures, 
understanding why can lead to a greater understanding of the underlying educational phenomena. Including measures of the learning processes thought to be responsible for the effects can be valuable for evaluating certain educational mechanisms responsible for observed outcomes. For example, large gains on final assessments can indicate a successful instructional intervention, intermediate measurements of the learning processes can provide direct evidence for mechanisms explaining why these interventions were successful.

In the event of a null outcome result, these process measures can be vital for interpreting whether the null result was due to an incorrect prediction or a weakness of the implementation. Each of the three examples of null results presented shows the importance of process measures. In investigating the general principle strategy, one weakness of the implementation was that the GP instruction only resulted in $28 \%$ of the students taking up the general principle strategy. This led researchers to hypothesize that differences between CVS and GPS as scientific inquiry strategies may not be fully clarified until instruction can support greater use of the strategies. In the algebra word problem study, investigating students' work and tendencies to use informal solution strategies helped to relate to previous research. In the force diagram study, prompting students to draw force diagrams had a null effect on accuracy, but did show a statistically significant difference in how it changed students' problem-solving process (i.e., finding an efficient shortcut). This combination of results showed that the process effects did not guarantee the overall accuracy effects, raising new questions about the combination of factors contributing to the original findings. In these cases, interpretations of a null result were enhanced with auxiliary measures, but even if the study findings are not a null result, including multiple measures in the research design can only provide additional evidence to add to the coherence of the interpretations and provide insight into the mechanisms by which the finding may have occurred.

When designing studies to extend prior research, attempts to replicate previous effects can indicate whether new null results are due to overall differences in implementation and context. For example, in the general principle study, the fact that the control-of-variables strategy showed benefits over no strategy confirmed that the research design and implementation was able to replicate previous findings. If this were not true, it would raise questions about the fidelity of how strategy instruction and measurement was conducted. Also, in our force diagram study, we sought to replicate Heckler's finding with an identical problem while also investigating whether the findings generalized to another force problem. Without the null result on the replicated problem, the null result on the new force problem could be incorrectly interpreted as solely due to changes in the problem type.
In evaluating results, we encourage researchers to look beyond statistical significance and novelty as the primary criterion evaluating the quality of quantitative research, both in their own work and the work of others. The alternative criteria we suggest are rooted in scientific coherence seeking. We suggest that researchers consider whether a study contributes to the development of coherent interpretations in the field. Successful replications of an effect can add additional evidence in favor of existing theories, and null results can contribute anomalous findings that spark new theoretical explanations and empirical efforts. Authors can aid this interpretation by highlighting the implications of their findings for advancing existing knowledge in the literature.

Another important criterion is that the research design and analysis should be high quality. To be clear, we are not suggesting that all null results should be published. Like Michelson and Morley's example, a publishable null result should be from a well-designed study with enough sensitivity that it could in principle detect an effect. For studies employing null hypothesis significance testing, this involves ensuring the study has enough statistical power (including a large enough sample size) to be able to detect an effect in principle. Regardless of null results, authors and reviewers should attend carefully to disciplinary guidelines $[19,20]$, such as including power analyses and aiming for close replication of methods that support the robustness of the null results. Additionally, authors and reviewers can attend to additional statistical descriptors such as effect size, which can describe the magnitude of effects for comparison with existing findings in the literature. Null results that challenge existing theories face the challenge of overturning existing, coherent explanations in the field. Establishing and communicating the rigor of the methodology is a key step, lest the surprising results be discarded as being anomalous.

\section{B. Current efforts to systematically combat bias against null results}

Of note, some of these practical directions are being implemented in systematic ways. As a way to explicitly combat null result bias, some journals have started taking explicit stances that the review process should focus on the quality of the research design and rigor of the analysis rather than the statistical significance or novelty of the results. For example, the multidisciplinary journal PLOS $O N E$ describes their criteria for publication and peer-review process as such:

"Often a journal's decision not to publish a paper reflects an editor's opinion about what is likely to have substantial impact in a given field. These subjective judgments can delay the publication of work that later proves to be of major significance. PLOS ONE will thoroughly peer-review your submissions and publish 
all papers that are judged to be technically rigorous. Judgments about the importance of any particular paper are then made after publication by the readership, who are the most qualified to determine what is of interest to them" [37].

In a parallel vein, other journals have been instituting the review of preregistered studies [38]. When preregistering a study, researchers specify the research questions, experimental design, and planned analyses beforehand. These journals review these registered reports before the study is conducted, with accepted research studies being promised publication independent of the findings. This approach emphasizes study design quality, the importance of the research question, and potential contribution to the literature. Without knowing whether the results will show statistically significant patterns, registered reports aim to reduce the publication bias against null results.

In addition, the growing practice of open science repositories can allow researchers to upload detailed information about their study, including detailed materials, raw data, and analysis methods, that will not fit within a published article. These study details can enhance discussions about data interpretation and the quality of replication studies. Less formally, journals such as PRPER publish supplemental materials that can also be used for this purpose if authors elect to invite readers to see the details of their study.

It is not known whether these initiatives will have the desired effect on the proportion of published null results in these fields, but they make available the data necessary to study their effectiveness in counteracting publication bias.

\section{CONCLUSION}

In this article, we point out the ways in which statistical null results can contribute to the broader scientific endeavor of advancing existing knowledge in PER. Null results, when situated in the existing literature and in a network of additional process measures, can be a useful tool for developing a scientific understanding of the phenomena of teaching and learning. The publication of null results from well-designed studies is more than just potentially useful; it is critical. In order to develop our understanding, we need a complete, unbiased sample of research results across which we can find patterns and reliable effects. In research disciplines that use null hypothesis significance testing, there unfortunately remains an ongoing bias against null results that threatens the validity of the research results. As a field that relies on methods and findings from these disciplines, PER runs the risk of inheriting this bias unless we examine this practice carefully.

We argue that learning how null results can contribute to the advancement of existing knowledge is a critical component of learning quantitative research methodology. Currently, this aspect of training in quantitative methods is lacking. While there have been attempts in various disciplines to offer guidelines to avoid over-reliance on p-value cutoff decisions, these guidelines still do not adequately address the potential contributions of null results. To that end, in this article we have made some practical suggestions for how to design, assess, and report the scientific contributions of null results, with an emphasis on seeking coherence with the established literature. These suggestions should not be viewed as a panacea for overcoming systematic and institutional biases against null results, but as first steps towards a more careful treatment of null results on the individual level. Our hope is these recommendations will help individual researchers avoid a bias against null results, and ultimately empower them to maintain a culture in PER of looking for results of scientific and instructional significance, regardless of their statistical significance.

\section{ACKNOWLEDGMENTS}

The inquiry strategies study was funded by NSF (09602 ) and The Hewlett Foundation. The word problem study was funded by IES (R305B150014).
[1] A. Franco, N. Malhotra, and G. Simonovits, Publication bias in the social sciences: Unlocking the file drawer, Science 345, 1502 (2014).

[2] J. Mervis, Why null results rarely see the light of day, Science 345, 992 (2014).

[3] D. Chavalaris, J. D. Wallach, A. H. T. Li, and J. P. Ioannidis, Evolution of reporting $\mathrm{p}$ values in the biomedical literature, 1990-2015, JAMA, J. Am. Med. Assoc. 315, 1141 (2016).

[4] A. Michelson and A. Morley, On the relative motion of the Earth and of the luminiferous ether, The Sidereal Messenger 6, 306 (1887).
[5] J. Cohen, The earth is round ( $p<.05)$, Am. Psychol. 49, 997 (1994).

[6] T. D. Sterling, W. L. Rosenbaum, and J. J. Weinkam, Publication decisions revisited: The effect of the outcome of statistical tests on the decision to publish and vice versa, Am. Statistician 49, 108 (1995).

[7] R. Rosenthal, The file drawer problem and tolerance for null results, Psychol. Bull. 86, 638 (1979).

[8] R. G. Smart, The importance of negative results in psychological research, Psychologie Canadienne 5, 225 (1964). 
[9] R. M. D. Smyth, J. J. Kirkham, A. Jacoby, D. G. Altman, C. Gamble, and P. R. Williamson, Frequency and reasons for outcome reporting bias in clinical trials: Interviews with trialists, Br. Med. J. 342, c7153 (2011).

[10] S. Freeman, S. L. Eddy, M. McDonough, M. K. Smith, N. Okoroafor, H. Jordt, and M. P. Wenderoth, Active learning increases student performance in science, engineering, and mathematics, Proc. Natl. Acad. Sci. U.S.A. 111, 8410 (2014).

[11] Open Science Collaborative, Estimating the reproducibility of psychological science, Science 349, aac4716 (2015).

[12] S. Wacholder, S. Chanock, M. Garcia-Closas, L. El Ghormli, and N. Rothman, Assessing the probability that a positive report is false: An approach for molecular epidemiology studies, J. Nat. Cancer Inst. 96, 434 (2004).

[13] B. A. Nosek, J. R. Spies, and M. Motyl, Scientific utopia: II. Restructuring incentives and practices to promote truth over publishability, Perspect. Psychol. Sci. 7, 615 (2012).

[14] J. W. Neuliep, Editorial bias against replication research, J. Social Behavior Personality 5, 85 (1990).

[15] G. N. Martin and R. M. Clarke, Are psychology journals anti-replication? A snapshot of editorial practices, Front. Psychol. 8, 523 (2017).

[16] R. J. Davis, Reproducibility Project: Cancer Biology, https://elifesciences.org/collections/9b1e83d1/ reproducibility-project-cancer-biology.

[17] R. E. Kirk, Practical significance: A concept whose time has come, Educ. Psychol. Meas. 56, 746 (1996).

[18] R. L. Rosnow and R. Rosenthal, Statistical procedures and the justification of knowledge in psychological science, Am. Psychol. 44, 1276 (1989).

[19] L. Wilkinson and the Task Force on Statistical Inference, Statistical methods in psychology journals: Guidelines and explanations, Am. Psychol. 54, 594 (1999).

[20] R. L. Wasserstein and N. A. Lazar, The ASA's statement on p-values: Context, process, and purpose, Am. Statistician 70, 129 (2016).

[21] R. R. Hake, Interactive-engagement vs traditional methods: A six thousand-student survey of mechanics test data for introductory physics courses, Am. J. Phys. 66, 64 (1998).

[22] M. Prince, Does active learning work? A review of the research, J. Eng. Ed. 93, 223 (2004).

[23] S. Freeman, S. L. Eddy, M. McDonough, M. K. Smith, N. Okoroafor, H. Jordt, and M. P. Wenderoth, Active learning increases student performance in science, engineering, and mathematics, Proc. Natl. Acad. Sci. U.S.A. 111, 8410 (2014).

[24] A. Madsen, S. B. McKagan, and E. C. Sayre, How physics instruction impacts students' beliefs about learning physics: A meta-analysis of 24 studies, Phys. Rev. ST Phys. Educ. Res. 11, 010115 (2015).
[25] S. Kanim and X. Cid, The demographics of physics education research, arXiv:1710.02598.

[26] L. D. Conlin, N. R. Hallinen, and D. L. Schwartz, Supporting middle schoolers' use of inquiry strategies for discovering multivariate relations in interactive physics simulations, in Proceedings of the International Conference of the Learning Sciences, Boulder, 2014, edited by J. L. Polman, E. A. Kyza, D. K. O'Neill, I. Tabak, W. R. Penuel, A. S. Jurow, K. O'Connor, T. Lee, and L. D'Amico (International Society of the Learning Sciences, Boulder, 2014), pp. 31-37.

[27] Z. Chen and D. Klahr, All other things being equal: Acquisition and transfer of the control of variables strategy, Child Development 70, 1098 (1999).

[28] D. Kuhn, Reasoning about multiple variables: Control of variables is not the only challenge, Sci. Ed. 91, 710 (2007).

[29] C. E. Wieman, W. K. Adams, and K. K. Perkins, PhET: Simulations that enhance learning, Science 322, 682 (2008).

[30] L. A. Milam, G. L. Cohen, C. Mueller, and A. Salles, Stereotype threat and working memory among surgical residents, Am. J. Surg. 216, 824 (2018).

[31] K. R. Koedinger and M. J. Nathan, The real story behind story problems: Effects of representations on quantitative reasoning, J. Learn. Sci. 13, 129 (2004).

[32] K. R. Koedinger, M. W. Alibali, and M. J. Nathan, Tradeoffs between grounded and abstract representations: Evidence from algebra problem solving, Cogn. Sci. 32, 366 (2008).

[33] E. Kuo, N. R. Hallinen, and L. D. Conlin, How prompting force diagrams discourages student use of adaptive problem-solving shortcuts, in Proceedings of the Physics Education Research Conference, College Park, 2015, edited by A. Churukian, D. Jones, and L. Ding (American Association of Physics Teachers, College Park, 2015).

[34] E. Kuo, N. R. Hallinen, and L. D. Conlin, When procedures discourage insight: epistemological consequences of prompting novice physics students to construct force diagrams, Int. J. Sci. Ed. 39, 814 (2017).

[35] A. F. Heckler, Some consequences of prompting novice physics students to construct force diagrams, Int. J. Sci. Ed. 32, 1829 (2010).

[36] P. Thagard, Explanatory coherence, Behavior Brain Sci. 12, 435 (1989).

[37] Journal Information, PLOS One, 2019, https://journals .plos.org/plosone/s/journal-information.

[38] C. D. Chambers, Z. Dienes, R. D. McIntosh, P. Rothstein, and K. Willmes, Registered reports: Realigning incentives in scientific publishing, Cortex 66, A1 (2015). 